\title{
Sellar Region
}

National Cancer Institute

\section{Source}

National Cancer Institute. Sellar Region. NCI Thesaurus. Code C155777.

The area inside or around the sella turcica. 